\title{
Pulling a folded polymer through a nanopore
}

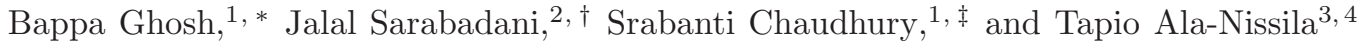 \\ ${ }^{1}$ Department of Chemistry, Indian Institute of Science Education and Research, Pune, Maharashtra, India. \\ ${ }^{2}$ School of Nano Science, Institute for Research in Fundamental Sciences (IPM), 19395-5531, Tehran, Iran \\ ${ }^{3}$ Department of Applied Physics and QTF Center of Excellence, \\ Aalto University School of Science, P.O. Box 11000, FI-00076 Aalto, Espoo, Finland. \\ ${ }^{4}$ Interdisciplinary Centre for Mathematical Modelling and Department of Mathematical Sciences, \\ Loughborough University, Loughborough, Leicestershire LE11 3TU, UK.
}

\begin{abstract}
We investigate the translocation dynamics of a folded linear polymer which is pulled through a nanopore by an external force. To this end, we generalize the iso-flux tension propagation (IFTP) theory for end-pulled polymer translocation to include the case of two segments of the folded polymer traversing simultaneously trough the pore. Our theory is extensively benchmarked with corresponding Molecular Dynamics (MD) simulations. The translocation process for a folded polymer can be divided into two main stages. In the first stage, both branches are traversing the pore and their dynamics is coupled. If the branches are not of equal length, there is a second stage where translocation of the shorter branch has been completed. Using the assumption of equal monomer flux of both branches, we analytically derive the equations of motion for both branches and characterise the translocation dynamics in detail from the average waiting time and its scaling form. Moreover, MD simulations are used to study additional details of translocation dynamics such as the translocation time distribution and individual monomer velocities.
\end{abstract}

The process of polymer translocation through nanometer sized pores plays an important role in many biological [1, 2] as well as technological applications [3, 4]. Experiments using single molecule precision have stimulated many theoretical and computational studies on polymer translocation [5 31]. Over the last few years a comprehensive theory of driven translocation dynamics has been developed based on the idea of tension propagation in the chain. This iso-flux tension propagation (IFTP) theory has been applied to a variety of different physical scenarios 22], including pore-driven translocation of flexible [18] and semi-flexible [21] polymers, end-pulled [20] polymers, and translocation of a flexible polymer through a flickering nanopore under an alternating external driving force acting in the pore [19].

Experiments on polymer translocation typically involve the electrically driven movement of charged polymers through pores whose typical diameters range from nanometers to tens of nanometers. In most of these experiments, the $\alpha$-hemolysin pore complex is used in common to study the translocation process [1, 5, 32. These experimental studies have been effective in distinguishing polymers of different molecular weights and sequences. However, there are several limitations in the use of this approach. The $\alpha$-hemolysin pore has a diameter about $2 \mathrm{~nm}$ such that only single-stranded DNA/RNA molecules or synthetic polyelectrolytes are restricted to thread through these protein channels. In the experiment by Kasionawicz et al., the ionic current through the voltage biased $\alpha$-hemolysin pore can detect the translocation

\footnotetext{
*These two authors contributed equally

${ }^{\dagger}$ These two authors contributed equally; Electronic address: jalal@ipm.ir

¥Electronic address: srabanti@iiserpune.ac.in
}

of single-stranded molecules through the narrow pore under the influence of an external field [5]. In addition, $\alpha$ hemolysin is not stable at wide experimental conditions such as high voltages ranges, temperatures, $\mathrm{pH}$ etc.

To overcome these difficulties, artificial solid-state nanopores have been developed and applied for studying polymer translocation. These nanopores can be tuned to larger diameters of $10-20 \mathrm{~nm}$ that allows translocation of double-stranded DNA molecules. Experiments on double-strand DNA translocation using silicon oxide nanopores have been reported by Dekker and coworkers $[33[36]$. Such synthetic pores have a lot of advantages over biological pores. For example, the synthetic pores are stable under experimental conditions such as high temperature, extreme voltage and $\mathrm{pH}$ values $37-$ 41]. Since solid-state pores can have larger diameters, it has been observed experimentally that a polymer can undergo not only single file motion through the pore but also in different folded states [42]. The formation of double-stranded DNA hairpins undergoing voltagedriven translocation through nanopores located in synthetic membranes has been studied using coarse-grained Langevin dynamics of translocation [43]. Kotsev and Kolomeisky have given a theoretical description of the translocation dynamics of polymer with folded configurations using simple discrete stochastic models 44]. The translocation dynamics is considered as the motion of the folded segment of the chain through the channel followed by the motion of the linear part of the polymer. However, driven polymer translocation is controlled by tension front propagation and a proper theoretical treatment using the IFTP theory has not been done to date.

To this end, here we study the translocation dynamics of a pulled folded polymer through a nanopore [20] by generalizing the IFTP theory to include the simultaneous translocation of two polymer strands in the pore. 

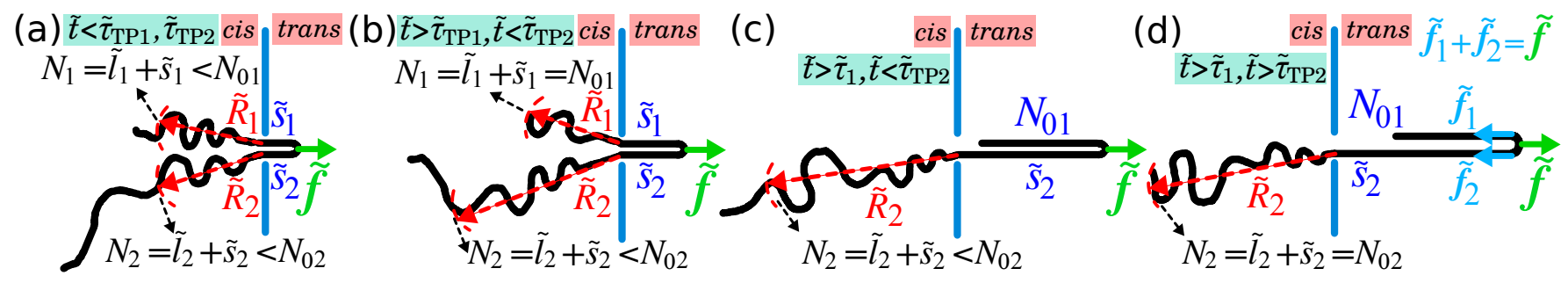

FIG. 1: (a) Schematic of the translocation process of a polymer with a folded configuration. The contour lengths for shorter and the longer branches are $N_{01}$ and $N_{02}$, respectively. The external driving force, $\tilde{f}$, acts on the pulled monomer that connects the shorter branch to the longer one. The direction of the force is from the cis towards the trans side. For both branches the translocation process is in the tension propagation (TP) stage, where the tension forces have not reached the branches ends and $N_{\mathrm{b}}=\tilde{l}_{\mathrm{b}}+\tilde{s}_{\mathrm{b}}<N_{0 \mathrm{~b}}$ with $\mathrm{b}=1$ and 2 denoting the branch $\mathrm{b}$ (TP1-TP2). For each branch the location of the tension front divides the mobile part from the immobile equilibrium part, and is represented by $\tilde{R}_{1}$ and $\tilde{R}_{2}$ for the shorter and longer branches, respectively. The number of translocated monomers (translocation coordinates) for shorter and longer branches are $\tilde{s}_{1}$ and $\tilde{s}_{2}$, respectively. (b) The translocation process is in TP stage for the longer branch $\left(N_{2}=\tilde{l}_{2}+\tilde{s}_{2}<N_{02}\right)$, while it is in post propagation (PP) stage for shorter one and $N_{1}=\tilde{l}_{1}+\tilde{s}_{1}=N_{01}$, where the tension force has already reached the shorter branch end (PP1-TP2). (c) The translocation process for the shorter branch has been completed while it is still in TP stage for the longer one $\left(\tau_{1}\right.$-TP2 stage, where $\tau_{1}$ stands for the translocation time of the shorter branch). (d) The same as panel (c) but the translocation process is in PP stage for the longer branch $\left(\tau_{1}\right.$-PP2). Here, the contribution of the total external driving force, $\tilde{f}$, to each branch has been shown as $\tilde{f}_{1}$ and $\tilde{f}_{2}$, and force balance for the pulled monomer gives $\tilde{f}_{1}+\tilde{f}_{2}=\tilde{f}($ not shown in panels (a)-(c)).

The theory is benchmarked with MD simulations. We consider both the symmetric case where the polymer is pulled in the middle monomer such that branches have equal lengths, and the asymmetric case with unequal branch lengths, as shown in Fig. 1. The details of the modified IFTP theory and MD simulations can be found in Secs. [I and [I] respectively, and the results are discussed in Sec. III Finally, Sec. IV is devoted to present the summary and conclusions.

\section{THEORETICAL MODEL}

In this section we generalize the IFTP theory for endpulled translocation dynamics to the present case of a folded linear polymer. When the contour lengths of both branches are the same, they traverse through the pore at equal rates on average. In contrast when the contour length of branches are not equal the translocation time of the shorter branch is less than that of the longer one. To develop the IFTP theory we consider here the high force limit and assume that the trans side sub-branches are fully straightened. This imposes the condition that the monomer flux which is the number of monomers that pass through the pore per unit time is the same for both branches. The dynamics of each branch is studied separately in the presence of the other one which leads to coupling between their equations of motion.

For the sake of simplicity, in the IFTP theory dimensionless units denoted by tilde are used as $\tilde{Y} \equiv Y / Y_{u}$, with the units of time $t_{u} \equiv \eta \sigma^{2} /\left(k_{\mathrm{B}} T\right)$, length $s_{u} \equiv \sigma$, velocity $v_{u} \equiv \sigma / t_{u}=k_{\mathrm{B}} T /(\eta \sigma)$, force $f_{u} \equiv k_{\mathrm{B}} T / \sigma$, monomer flux $\phi_{u} \equiv k_{\mathrm{B}} T /\left(\eta \sigma^{2}\right)$ and friction $\Gamma_{u} \equiv \eta$, where $T$ is the temperature of the system, $k_{\mathrm{B}}$ is the Boltzmann constant, $\sigma$ is the length of each segment, and the solvent friction per monomer is $\eta$. The quantities without the tilde are expressed in the Lennard-Jones units.

In Fig. 1 we show a schematic of the translocation process of a folded polymer. The contour lengths for short and the long branches are denoted by $N_{01}$ and $N_{02}$, respectively, i.e. $N_{01} \leq N_{02}$. The external driving force, $\tilde{f}$, acts on the monomer which connects the short branch to the long one, and its direction is from cis towards the trans side. Panel (a) illustrates the tension propagation (TP) stage for both branches (TP1-TP2), wherein the tension force has not reached the branches' ends and $N_{\mathrm{b}}=\tilde{l}_{\mathrm{b}}+\tilde{s}_{\mathrm{b}}<N_{0 \mathrm{~b}}(\mathrm{~b}=1$ and 2 stand for short and long branches, respectively). $N_{\mathrm{b}}$ is the total number of beads in branch $b$ that have been already affected by the tension force, and $\tilde{l}_{\mathrm{b}}$ is the number of beads in the mobile domain of the cis side sub-branch b. In the TP stage the location of the tension front separates the mobile sub-branches from the immobile equilibrium ones, and is represented by $\tilde{R}_{\mathrm{b}}$. During the TP stage it is assumed that $\tilde{R}_{1}=\tilde{R}_{2}$. The number of translocated monomers (translocation coordinates) for shorter and longer branches are $\tilde{s}_{1}$ and $\tilde{s}_{2}$, respectively. When both branches are inside the pore then $\tilde{s}_{1}=\tilde{s}_{2}$. This is due to the strong force limit, wherein both sub-branches in the trans side are straightened. As time passes the shorter branch experiences the post propagation (PP1) stage, where the tension force has reached its end, and $N_{1}=\tilde{l}_{1}+\tilde{s}_{1}=N_{01}$, while the longer one is still in the TP stage (PP1-TP2). This is illustrated in panel (b). Then as can be seen in panel (c), the translocation process for the short branch has been completed while it is 
still in TP stage for the long one $\left(\tau_{1}-\mathrm{TP} 2\right.$, where $\tau_{1}$ is the translocation time for the shorter branch). Finally, panel (d) shows that the translocation process is in PP stage for the long branch $\left(\tau_{1}\right.$-PP2). Moreover, in panel (d), the contribution of the total external driving force, $\tilde{f}$, to each branch is illustrated as $\tilde{f}_{1}$ and $\tilde{f}_{2}$. The force balance for the pulled monomer gives $\tilde{f}_{1}+\tilde{f}_{2}=\tilde{f}$ (not shown in panels (a)-(c)).

In addition, in the very strong force limit of $\tilde{f}_{0 \mathrm{~b}}>\tilde{N}_{\mathrm{b}}$ the mobile sub-branch $\mathrm{b}$ in the cis side is fully straightened (strong stretching (SSC) limit). In the moderate external force limit of $1<\tilde{f}_{0 \mathrm{~b}}<\tilde{N}_{\mathrm{b}}$ the regime is called stem-flower (SFC) as the shape of the mobile sub-branch $\mathrm{b}$ in the cis side is similar to a stem followed by a flower. Finally, the weak force limit of $\tilde{f}_{0 \mathrm{~b}}<1$ is called the trumpet (TRC) regime, where the mobile sub-branch in the cis side assumes a trumpet shape.

As mentioned above and depicted in Fig. 1 only the SS regime in the trans side is considered here, and therefore using the deterministic version of the iso-flux Brownian dynamics tension propagation theory without any entropic force [13, 18] is a very good approximation. Within this framework, the equation of motion for the time evolution of the translocation coordinate $\tilde{s}_{\mathrm{b}}$ for branch $\mathrm{b}$, that is the number of translocated beads to the trans side, is written as

$$
\tilde{\Gamma}_{\mathrm{b}}(\tilde{t}) \frac{\mathrm{d} \tilde{s}_{\mathrm{b}}}{\mathrm{d} \tilde{t}}=\tilde{f}_{\mathrm{b}}
$$

where $\tilde{\Gamma}_{\mathrm{b}}(\tilde{t})$ is the effective friction and $\tilde{f}_{\mathrm{b}}$ is the contribution of the total external driving force $\tilde{f}$ to branch $\mathrm{b}$ as depicted in Fig. 1(d). According to Refs. 20, 21] the effective friction can be obtained as $\tilde{\Gamma}_{\mathrm{b}}(\tilde{t})=\tilde{\eta}_{c i s \mathrm{~b}}(\tilde{t})+$ $\tilde{\eta}_{\mathrm{pj}}(\tilde{t})+\tilde{\eta}_{\mathrm{TSb}}(\tilde{t})$, where $\tilde{\eta}_{c i s \mathrm{~b}}(\tilde{t})$ denotes the friction due the cis side mobile sub-branch $\mathrm{b}$ in the solvent, $\tilde{\eta}_{\mathrm{pj}}(\tilde{t})$ is the pore friction, and $\tilde{\eta}_{\mathrm{TSb}}(\tilde{t})$ presents the friction due the movement of the trans side mobile sub-branch $b$. When both branches are inside the pore, i.e. in the stages TP1$\mathrm{TP} 2$, or PP1-TP2, $\mathrm{j}=12$ and when only the longer one is inside the pore, i.e. in the stages $\tau_{1}$-TP2 or $\tau_{1}$-PP2, $\mathrm{j}=2$. It should be mentioned that the trans side friction terms play an important role in the dynamics of the current system [20, 21]. This will be discussed in detail below. Later it will be shown how to find the values of $\tilde{f}_{1}$ and $\tilde{f}_{2}$ during the translocation process. In the symmetric case when the contour lengths of both branches are the same, i.e. $N_{01}=N_{02}$, then due to the symmetry of the system $\tilde{f}_{1}=\tilde{f}_{2}=\tilde{f} / 2$ during the whole translocation process.

Using the IFTP theory the dynamics of each branch in the cis and in the trans sides is separately solved with the corresponding TP equations. The iso-flux (IF) approximation is used to find the TP equations [45]. In the IF approximation the monomer flux $\tilde{\phi}(\tilde{t})=\mathrm{d} \tilde{s} / \mathrm{d} \tilde{t}$ within the mobile domain for each branch is constant in space but evolves with time. In the TP1-TP2 and PP1-TP2 stages the tension front is located at distance $\tilde{x}=\tilde{R}_{\mathrm{b}}(\tilde{t})$ to the pore in the cis side. Inside each branch, the tension force is mediated from the pulled monomer at the distance $\tilde{s}$ in the trans side all the way to the pore located at $\tilde{x}=0$ and then to the last mobile bead $N_{\mathrm{b}}$ located in the tension front in the cis side. Performing the integration of the local force-balance relation $\mathrm{d} \tilde{f}_{\mathrm{b}}\left(\tilde{x}^{\prime}\right)=-\tilde{\phi}_{\mathrm{b}}(\tilde{t}) \mathrm{d} \tilde{x}^{\prime}$ [18, 20] over the distance from the location of the pulled monomer to $\tilde{x}$, gives the tension force at the distance $\tilde{x}$ as

$$
\tilde{f}_{\mathrm{b}}(\tilde{x}, \tilde{t})=\tilde{f}_{0 \mathrm{~b}}-\tilde{x} \tilde{\phi}_{\mathrm{b}}(\tilde{t}),
$$

where $\tilde{f}_{0 \mathrm{~b}}=\tilde{f}_{\mathrm{b}}-\tilde{\eta}_{\mathrm{pj}} \tilde{\phi}_{\mathrm{b}}(\tilde{t})-\tilde{\eta}_{\mathrm{TSb}} \tilde{\phi}_{\mathrm{b}}(\tilde{t})$ is the force at the entrance of the pore in the cis side. Combining Eq. (2) and the fact that the tension force vanishes at the tension front, i.e. $\tilde{f}_{\mathrm{b}}\left(\tilde{R}_{\mathrm{b}}, \tilde{t}\right)=0$ yields the monomer flux as

$$
\tilde{\phi}_{\mathrm{b}}(\tilde{t})=\frac{\tilde{f}_{\mathrm{b}}}{\tilde{R}_{\mathrm{b}}+\tilde{\eta}_{\mathrm{pj}}+\tilde{\eta}_{\mathrm{TSb}}} .
$$

Since we are in the SS limit for both sub-branches in the trans side, $\tilde{\eta}_{\mathrm{TSb}}=\tilde{s}_{\mathrm{b}}=\tilde{s}$. Moreover, when both branches are inside the pore $\tilde{\eta}_{\mathrm{pj}}=\tilde{\eta}_{\mathrm{p} 12}$, and if only the long branch is located in the pore $\tilde{\eta}_{\mathrm{pj}}=\tilde{\eta}_{\mathrm{p} 2}$.

To determine $\tilde{f}_{1}$ and $\tilde{f}_{2}$, two equations must be solved. The first equation is $\tilde{f}_{1}+\tilde{f}_{2}=\tilde{f}$, which is the force balance equation for the pulled monomer as shown in Fig. 1(d). The second one comes from the fact that

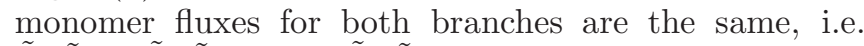
$\tilde{\phi}_{1}(\tilde{t})=\tilde{\phi}_{2}(\tilde{t})$, where $\tilde{\phi}_{\mathrm{b}}(\tilde{t})$ has been defined in Eq. (3). Solving these two equations gives

$$
\begin{aligned}
& \tilde{f}_{1}=\tilde{f} \times\left(1+\frac{\tilde{R}_{2}+\tilde{\eta}_{\mathrm{TS} 2}+\tilde{\eta}_{\mathrm{p} 12}}{\tilde{R}_{1}+\tilde{\eta}_{\mathrm{TS} 1}+\tilde{\eta}_{\mathrm{p} 12}}\right)^{-1} ; \\
& \tilde{f}_{2}=\tilde{f} \times\left(1+\frac{\tilde{R}_{1}+\tilde{\eta}_{\mathrm{TS} 1}+\tilde{\eta}_{\mathrm{p} 12}}{\tilde{R}_{2}+\tilde{\eta}_{\mathrm{TS} 2}+\tilde{\eta}_{\mathrm{p} 12}}\right)^{-1} .
\end{aligned}
$$

Inserting the above $\tilde{f}_{\mathrm{b}}$ into Eq. (3) , the monomer flux reads as

$$
\tilde{\phi}_{1}(\tilde{t})=\tilde{\phi}_{2}(\tilde{t})=\tilde{\phi}(\tilde{t})=\frac{\tilde{f}}{\tilde{R}_{1}+\tilde{R}_{2}+2 \tilde{\eta}_{\mathrm{p} 12}+\tilde{\eta}_{\mathrm{TS} 1}+\tilde{\eta}_{\mathrm{TS} 2}} .
$$

If the contour lengths for both branches are the same, or the translocation process is in TP1-TP2, then due to the symmetry $\tilde{R}_{1}=\tilde{R}_{2}$. This leads to $\tilde{f}_{1}=\tilde{f}_{2}=\tilde{f} / 2$, and consequently $\tilde{\phi}(\tilde{t})=(1 / 2) \tilde{f} \times\left(\tilde{R}+\tilde{\eta}_{\mathrm{p} 12}+\tilde{s}\right)^{-1}$.

In the $\tau_{1}$-TP2 and $\tau_{1}$-PP2 stages where the whole short branch has been translocated to the trans side, the time evolution of $\tilde{s}_{2}$ is given by Eq. (1) with index $\mathrm{b}=2$, and similar procedure to the TP1-TP2 and PP1-TP2 stages is employed to obtain the monomer flux as

$$
\tilde{\phi}(\tilde{t})=\frac{\tilde{f}}{\tilde{R}_{2}+\tilde{\eta}_{\mathrm{p} 2}+\tilde{\eta}_{\mathrm{TS} 2}+N_{01}},
$$

where $\tilde{\eta}_{\mathrm{p} 2}$ is the pore friction when only the long branch is inside the pore, $\tilde{\eta}_{\mathrm{TS} 2}=\tilde{s}_{2}$, and $N_{01}$ is the trans side friction due to the whole mobile short branch. 
In the TP1-TP2 and PP1-TP2 stages combining Eqs. (11) and (3) and (41), the time evolution of the translocation coordinates are obtained for short and long branches provided that the time evolution of the location of the tension front for each branch is known. On the other hand in the $\tau_{1}$-TP2 and $\tau_{1}$-PP2 stages Eq. (11) together with (6) give the translocation coordinate for the longer branch as a function of time again if the location of the tension front for the long branch is know. Therefore, to proceed further the time evolution of the location of the tension fronts for the short as well as long branches, $\tilde{R}_{1}$ and $\tilde{R}_{2}$, should be obtained.

To obtain the time evolution of $\tilde{R}_{\mathrm{b}}$ in the TP stage one can use the end-to-end distance $\tilde{R}_{\mathrm{b}}=A_{\nu} N_{\mathrm{b}}^{\nu}$, where $A_{\nu}=1.15$ is constant obtained from MD simulations, $N_{\mathrm{b}}=\tilde{l}_{\mathrm{b}}+\tilde{s}_{\mathrm{b}}$, and $\nu=0.5888$ is the Flory exponent for $3 \mathrm{D}$. The time derivative of the above relation is then written as $\dot{\tilde{R}}_{\mathrm{b}}=A_{\nu}^{1 / \nu} \tilde{R}_{\mathrm{b}}^{(\nu-1) / \nu}\left(\dot{\tilde{l}}_{\mathrm{b}}+\dot{\tilde{s}}_{\mathrm{b}}\right)$, where $\dot{\tilde{s}}_{\mathrm{b}}$ is the monomer flux and $\dot{\tilde{l}}_{\mathrm{b}}$ must be found. In the $\mathrm{SSC}$ regime as the mobile sub-branch $\mathrm{b}$ in the cis side is fully straightened the monomer number density is unity, while according to the blob theory in the SFC and TRC regimes the monomer number density, which is $\tilde{\sigma}_{\mathrm{b}}(\tilde{x})=\left|\tilde{f}_{\mathrm{b}}(\tilde{x})\right|^{(\nu-1) / \nu}$, is larger than unity due to the folding of the chain. By integrating $\tilde{\sigma}_{\mathrm{b}}^{\mathrm{I}}(\tilde{x})(\mathrm{I}=\mathrm{SSC}, \mathrm{SFC}$ or TRC) over the distance from the pore entrance in the cis side to the location of the tension front, $\tilde{l}_{\mathrm{b}}^{\mathrm{I}}$ is obtained as

$$
\begin{aligned}
\tilde{l}_{\mathrm{b}}^{\mathrm{SSC}} & =\tilde{R}_{\mathrm{b}} \\
\tilde{l}_{\mathrm{b}}^{\mathrm{SFC}} & =\tilde{R}_{\mathrm{b}}+\frac{1-\nu}{2 \nu-1} \frac{1}{\tilde{\phi}_{\mathrm{b}}} ; \\
\tilde{l}_{\mathrm{b}}^{\mathrm{TRC}} & =\frac{\nu}{2 \nu-1} \tilde{R}_{\mathrm{b}}^{(2 \nu-1) / \nu} \tilde{\phi}_{\mathrm{b}}^{(\nu-1) / \nu} .
\end{aligned}
$$

Combining the time derivative of $\tilde{l}_{\mathrm{b}}^{\mathrm{I}}$ with the above relation for $\dot{\tilde{R}}_{\mathrm{b}}$, the equations of motion for the location of the tension front when both branches are inside the pore are obtained as

$$
\dot{\tilde{R}}_{1}^{\mathrm{I}, \mathrm{J}, 12}=\mathcal{U}_{1}^{\mathrm{I}, \mathrm{J}} ; \quad \dot{\tilde{R}}_{2}^{\mathrm{I}, \mathrm{J}, 12}=\mathcal{U}_{2}^{\mathrm{I}, \mathrm{J}},
$$

and when only the longer branch remains inside the pore

$$
\dot{\tilde{R}}_{2}^{\mathrm{I}, \mathrm{J}, 2}=\mathcal{V}_{2}^{\mathrm{I}, \mathrm{J}}
$$

where I denotes the different regimes of SSC, SFC and TRC, J stands for different stages of TP and PP, superscript 12 in the left hand side of Eq. (8) means that both the short and the long branches are inside the pore, and superscript 2 in the left hand side of Eq. (9) means that only the long branch is inside the pore. $\tilde{\mathcal{U}}_{\mathrm{b}}^{\mathrm{I}, \mathrm{J}}$ and $\tilde{\mathcal{V}}_{2}^{\mathrm{I}, \mathrm{J}}$ are functions of $\nu, A_{\nu}, \tilde{R}_{2}, \tilde{f}$, and $\tilde{\phi}$, and their explicit forms can be found in Appendix A.

To have the full solution of the IFTP model when both branches are passing through the pore Eqs. (11), (5) and (8) should be solved self-consistently, and when only the long branch remains one must solve Eqs. (19), (6) and (9).

\section{MOLECULAR DYNAMICS SIMULATIONS}

To examine the validity of the theory we have performed extensive molecular dynamics (MD) simulations of a folded polymer pulled through a pore by the monomer separating the two folded branches with $N_{01} \leq N_{02}$ monomers. We have employed Langevin dynamics simulations using the LAMMPS package [46]. The polymer chain is modeled as a coarse-grained selfavoiding bead-spring chain, with each bead representing a monomer. The successive beads are connected by the finitely extensible nonlinear elastic (FENE) spring interaction given by :

$$
U_{\mathrm{FENE}}(r)=-\frac{k R_{0}^{2}}{2} \ln \left(1-\frac{r^{2}}{R_{0}^{2}}\right)
$$

where $k$ is the spring constant and $R_{0}$ is the maximum bond length. The excluded volume interaction between any two beads, and between a polymer bead and the pore particles is given by a repulsive Lenard-Johns (LJ) interaction

$$
\begin{aligned}
& U_{\mathrm{LJ}}(r)=4 \epsilon\left[\left(\frac{\sigma}{r}\right)^{12}-\left(\frac{\sigma}{r}\right)^{6}\right]+\epsilon: \text { if } r \leq r_{\mathrm{c}}, \\
& =0 \quad: \text { if } r \geq r_{\mathrm{c}},
\end{aligned}
$$

where $r$ is the distance between two beads, $\epsilon$ is the interaction strength and $\sigma$ is the diameter of each bead. The cut-off radius is $r_{\mathrm{c}}=2^{1 / 6} \sigma$. The Langevin equation for each $i^{\text {th }}$ particle of the system is solved as

$$
m \ddot{r}_{i}=-\nabla\left(U_{\mathrm{LJ}}+U_{\mathrm{FENE}}\right)+f+F_{i}^{F}+F_{i}^{R}
$$

where each polymer bead experiences conservative, frictional and random forces. The frictional force $F_{i}^{F}=$ $-\eta v_{i}, v_{i}$ is the monomer velocity, $\eta$ is the solvent friction coefficient, $f$ is the external pulling force acting on the monomer being pulled, and $F_{i}^{R}$ is the random force with zero mean $\left\langle F_{i}^{R}(t)\right\rangle=0$ which satisfies the fluctuationdissipation theorem $\left\langle F_{i}^{R}(t) F_{j}^{R}\left(t^{\prime}\right)\right\rangle=6 \eta k_{\mathrm{B}} T \delta_{i j} \delta\left(t-t^{\prime}\right)$. The parameters of our MD simulations in LJ units have been chosen as $\sigma=1, \epsilon=1, R_{0}=1.5 \sigma, k=30$ and $\eta=0.7$. Here the external driving force is chosen as $f=100$, and $k_{\mathrm{B}} T=1.2$. In our model, the mass of each bead $m$ is about $936 \mathrm{amu}$, its size $\sigma$ corresponds approximately to the Kuhn length of a single-stranded DNA, and the interaction strength $\epsilon$ is $3.39 \times 10^{21} \mathrm{~J}$ at room temperature $(T=295 \mathrm{~K})$. In LJ units, the time and force scales are $32.1 \mathrm{ps}$ and $2.3 \mathrm{pN}$, respectively [13, 18]. The time step for the integration of the Langevin equation has been chosen as $\mathrm{d} t=0.005$ (in LJ units) during the equilibration of the system and 0.0005 for the actual translocation process. It should be mentioned that the width of the pore is small and only one monomer for a linear chain or two monomers for a folded polymer can be inside the nanopore.

As schematically shown in Fig. 1. we consider a chain of an odd number of beads, $N_{0}=101$ with the pulled bead 
connecting the two branches placed at the pore. Here we consider folded chains with three different branch lengths of 51:51, 31:71 and 11:91. At the beginning of the simulations the folded polymer is carefully equilibrated with the pulled bead fixed at the pore. Then the constraint is removed and the external pulling force $f$ starts acting on the bead from cis towards the trans side. As mentioned in the theory section the pulling force is strong enough such that the chain is essentially straightened on the trans side. Translocation time is recorded separately for the short and long segments. Our MD data here have been averaged over $400-500$ independent runs.

\section{RESULTS}

\section{A. Waiting time distribution}

In order to examine the validity of the IFTP theory we first compare dynamics of the translocation process at the monomer level between IFTP theory and MD simulations using the waiting time distribution (WT), which is the average time that each bead spends in the pore during the process of the translocation. In Fig. 2 we show our data for the three sets of folded polymers. In panel (a) the WT $w(\tilde{s})$ has been plotted as a function of the translocation coordinate $\tilde{s}$ for the case wherein the branches have the same contour length, i.e. $N_{01}=N_{02}=51$, with the total contour length of linear polymer $N_{0}=N_{01}+N_{02}=101$. Here the bead that connects the two branches naturally belongs to both of them. The pore friction used in the IFTP theory is $\eta_{\mathrm{p} 12}=6$, external driving force which acts on the connecting bead is $f=100$ (both in the theory as well as in the MD simulations) and $k=30$ is the spring constant in the bead-spring model used in the MD simulations. Open light blue circles and open orange triangles are MD data while the solid red line represents the IFTP theory results. Panels (b) and (c) are the same as (a) but for different values of the contour lengths of the two branches $N_{01}=31$ and $N_{02}=71$, and $N_{01}=11$ and $N_{02}=91$, respectively, with constant $N_{0}=101$. In panels (b) and (c) when both branches are traversing the pore, the pore friction in the IFTP theory is chosen as $\eta_{\mathrm{p} 12}=6$, and after the translocation of the short branch, it reduces to the fixed value of $\eta_{\mathrm{p} 2}=3$. Open light blue circles and open orange triangles are MD data for short and long branches, respectively, while the solid blue and the dashed red lines represent the IFTP theory results for the short and long branches, respectively. It is obvious from the figure that the agreement between IFTP theory and MD simulations is very good.

\section{B. Translocation time for each branch}

The central quantity that describes the global dynamics of the polymer translocation through a nanopore is the average translocation time $\tilde{\tau}$. To find the IFTP translocation time for the short and long branches, $\tau_{1}$ and $\tau_{2}$, respectively, Eqs. (1), (5), (6), (8) and (9) must be solved self-consistently. To compare with MD, in Fig. 3 the normalized translocation times for the short branch $\tau_{1} / \tau_{1}\left(N_{01}=51\right)$ has been plotted as a function of the contour length of the short branch $N_{01}$ (left and bottom blue axes), while the normalized translocation time for the long branch $\tau_{2} / \tau_{2}\left(N_{02}=51\right)$, has been plotted as a function of the contour length of the longer branch $N_{02}$ (right and top red axes). $\tau_{1}\left(N_{01}=51\right)=\tau_{2}\left(N_{02}=51\right)$ are the translocation times for the two branches of a folded linear polymer with contour length of $N_{0}=101$ when the polymer is folded in the middle. For the short branch the light blue open circles present the MD data, and the solid blue line shows the IFTP theory results. On the other hand the open orange triangles and the solid red line present the MD and the IFTP theory results, respectively, for the long branch. As the figure shows the data are in excellent agreement as already expected from the WT distributions.

\section{Scaling of the translocation time}

To find a scaling form for the translocation time for the short branch Eq. (5) should be integrated over $N_{1}$ from zero to $N_{01}$ in the TP1-TP2 stage as depicted in Fig. 11(a) followed by the integration of $\tilde{R}_{1}$ from $\tilde{R}_{1}\left(N_{01}\right)$ to zero in the PP1-TP2 stage as shown in Fig. 1 (b). This yields the translocation time for the short branch in the SS regime for the cis and trans sides as

$$
\tilde{\tau}_{1}=\frac{1}{\tilde{f} / 2}\left(\frac{A_{\nu}}{1+\nu} N_{01}^{1+\nu}+\frac{N_{01}^{2}}{2}+\tilde{\eta}_{\mathrm{p} 12} N_{01}\right),
$$

where the first, second and the third terms in the r.h.s of Eq. (13) are due to the mobile sub-branch friction in the cis side, straightened sub-chain friction in the trans side and the pore friction, respectively. Similarly, to find a closed form of the translocation time for the longer branch in the SS regime, one needs to integrate Eq. (6) over $N_{2}$ from $N_{01}$ to $N_{02}$ in the $\tau_{1}$-TP2 stage as presented in Fig. 1(c) followed by the integration of $\tilde{R}_{2}$ from $\tilde{R}_{2}\left(N_{02}\right)$ to zero in the $\tau_{1}$-PP2 stage as shown in Fig.11(d). Following this procedure the translocation time for the longer branch in the SS regime for the cis and trans sides can be written as

$$
\begin{aligned}
\tilde{\tau}_{2}=\frac{1}{\tilde{f}}[ & +\frac{A_{\nu}}{1+\nu} N_{01}^{1+\nu}+\frac{A_{\nu}}{1+\nu} N_{02}^{1+\nu}+\frac{N_{02}^{2}}{2}-\frac{N_{01}^{2}}{2} \\
& \left.+2 \tilde{\eta}_{\mathrm{p} 12} N_{01}+\tilde{\eta}_{\mathrm{p} 2}\left(N_{02}-N_{01}\right)+N_{01} N_{02}\right]
\end{aligned}
$$

revealing how the contour lengths of the short and long branches are coupled to each other. In Fig. 3 the results from the Eqs. (13) and (14) are shown in light blue and yellow dashed lines for the short and long branches, respectively. As can be seen there is a very good agreement 


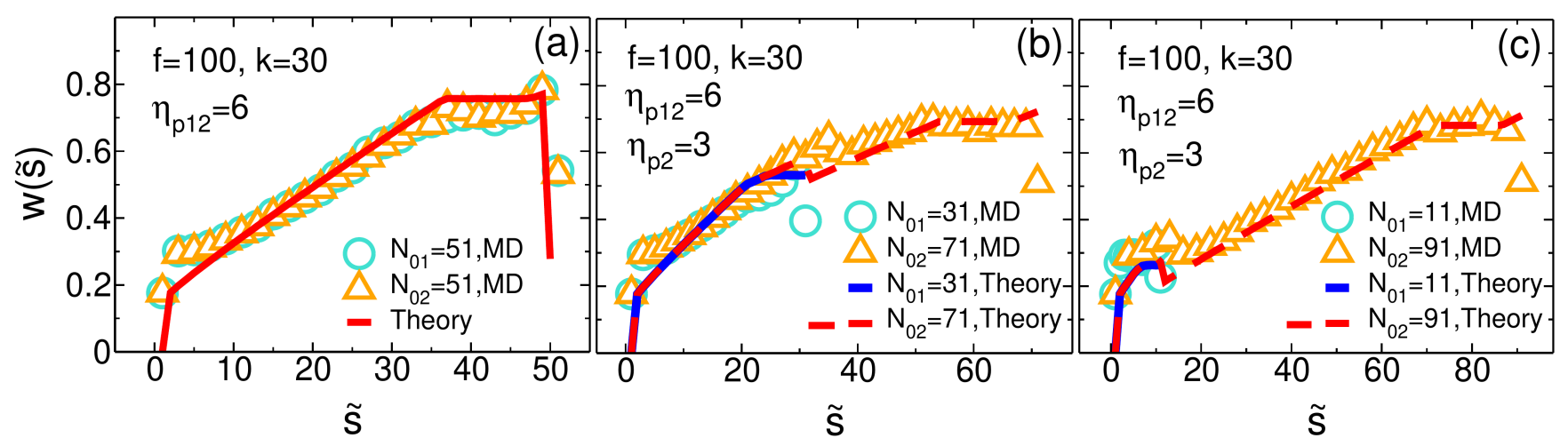

FIG. 2: (a) The waiting time distribution $w(\tilde{s})$ as a function of the translocation coordinate $\tilde{s}$ for the folded linear polymer chain with equal branches, i.e. $N_{01}=N_{02}=50+1$, with $N_{0}=N_{01}+N_{02}=101$, pore friction $\eta_{\mathrm{p} 12}=6$, external driving force $f=100$ and spring constant $k=30$ in the bead-spring model used in the MD simulations. Open light blue circles and open orange triangles are MD data while the solid red line represents the IFTP theory results. Panels (b) and (c) are the same as panel (a) but for different values of the contour lengths of the two branches $N_{01}=31$ and $N_{02}=71$, and $N_{01}=11$ and $N_{02}=91$, respectively, with constant $N_{0}=101$. In panels (b) and (c) when both branches are traversing the pore, the pore friction in the theory is $\eta_{\mathrm{p} 12}=6$, and after the translocation of the shorter branch to the cis side it reduces to the fixed value of $\eta_{\mathrm{p} 2}=3$. Open light blue circles and open orange triangles are MD data for short and long branches, respectively, while the solid blue and the dashed red lines represent the IFTP theory results for the short and long branches, respectively.

between the normalized scaling formula, the MD results and the IFTP theory, although the scaling formulae in Eqs. (13) and (14) have been obtained in the limit of long branches in the SS regime.

\section{Monomer velocities}

In this subsection we present additional data for the monomer velocities from the MD simulations (averaged over 400-500 successful translocation runs). In Fig. 4(a) the velocities of the individual monomers for the polymer branches, $v(\mathrm{~m})$, have been plotted as a function of the normalized monomer index, $m / N_{02}$, at different moments of the translocation process $t=2-20$ for the symmetric folded chain with $N_{01}=N_{02}=51$. The solid lines present the monomer velocities of the branch 1, while open symbols show the velocities for the branch 2. Panels (b) and (c) are the same as panel (a) but for an asymmetric folded polymer chain with branch contour lengths $N_{01}=31$ and $N_{02}=71$, and $N_{01}=11$ and $N_{02}=91$, respectively. In panel (b) the data is presented during $t=2-28$ and in panel (c) during $t=1-36$. Here, the individual monomer velocities have been averaged in the direction of the driving force, i.e. horizontal direction from the cis to the trans side. As can be seen in panel (a) for the symmetric 51:51 folded chain, the monomer velocities for both branches are the same, and the tension propagation occurs in an identical manner for both branches. At each moment, the velocities of the monomers for both branches that have already moved to the trans side have the same and constant value due to the strong pulling force. For the monomer beads which are in the cis side, there is a drop in velocity during the
TP1-TP2 stage along both the chain branches and the velocity is zero for the non-mobile equilibrium part of the branches. In panels (b) and (c) as time passes, the TP1 of the short branch $N_{01}$ ends and the PP1 stage starts. For example in panel (b) the interval $5<t<15$ is the transient window from TP1 to PP1 stage for the short branch, while the long one still is in its TP2 stage and the tension is still propagating along its backbone on the cis side. In the PP2 stage, as time progresses, the monomers' velocity of the long branch on the cis side subbranch increases and finally becomes equal to that of the trans side sub-branch. Moreover, in all three panels (a), (b) and (c) the velocities of the individual monomers of both branches coincide with each other. This tells us that the equal monomer flux assumption in the IFTP theory is correct.

\section{E. Translocation time distribution}

Finally, the probability distributions of the translocation time, $P(\tau)$, for the folded polymer with various branched contour lengths are investigated using MD simulations. In panel (a) of Fig. 5 the probability distribution of the translocation time for both branches of the folded polymer chain with contour length of $N_{0}=101$, has been plotted as a function of the translocation time $\tau$ when the folding is symmetric and the contour lengths of both branches are the same as $N_{01}=N_{02}=51$. Panels (b) and (c) are the same as panel (a) but for different sets for the contour lengths of the beaches $N_{01}=31$ and $N_{02}=71$, and $N_{01}=11$ and $N_{02}=91$, respectively. When the folded chain becomes more asymmetrical, i.e. the contour length of one branch gets shorter and in con- 


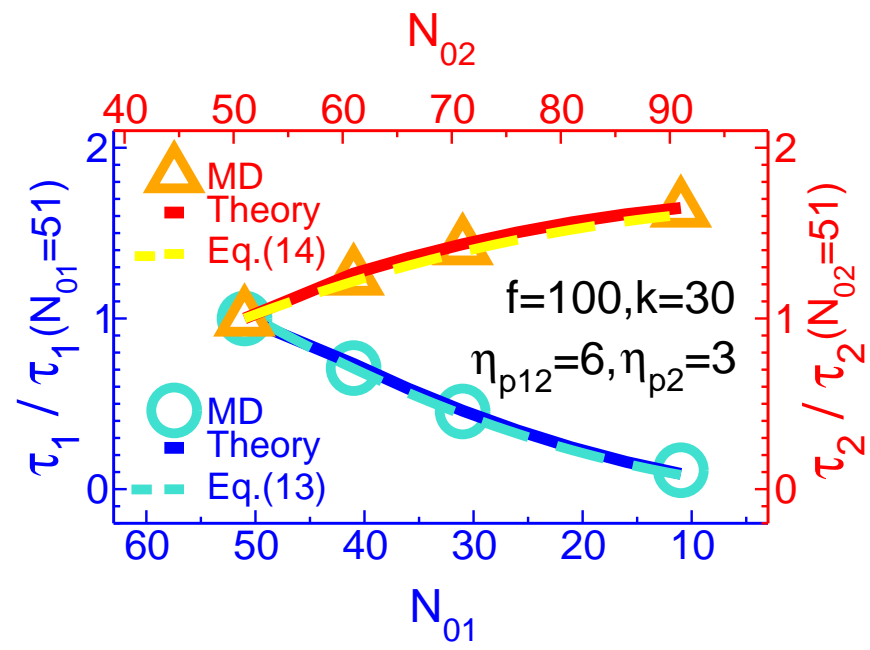

FIG. 3: Normalized translocation time $\tau_{1} / \tau_{1}\left(N_{01}=51\right)$ as a function of the contour length for the short branch $N_{01}$, and $\tau_{2} / \tau_{2}\left(N_{02}=51\right)$ as a function of the contour length for the long branch $N_{02}$. For the short branch the light blue open circles present the MD data, and the solid blue line shows the IFTP theory results. For the long branch the open orange triangles and the solid red line present the MD and IFTP theory results, respectively. The data for the short branch must be read from the bottom horizontal and left vertical blue axes, while for the long branch the data should be read from the top horizontal and right vertical red axes. The results of the Eqs. (13) and (14) are shown in light blue dashed and yellow dashed lines for the short and long branches, respectively.

trast the other one becomes longer, the probability distributions for different branches are separated more from each other. As can be seen in Fig. 5. (c) the separation is more pronounced than panel (b). Moreover, for shorter branch the width of the probability distribution is narrower due to the decrease in the spatial fluctuations of the branch configurations.

\section{SUMMARY AND CONCLUSION}

In this work, we have theoretically and computationally studied the translocation dynamics of a singly-folded polymer chain pulled through a nanopore by applying a pulling force on the monomer connecting the two branches. The pulling force initiates a tension front that propagates though the folds during translocation. To properly treat this, we have generalized the IFTP theory to the present case. We have also performed extensive MD simulations of a coarse-grained bead-spring model to benchmark the theory. The WT distribution obtained from the IFTP theory has been compared with MD one showing good agreement at the monomer level dynamics. Then, the global dynamics of the translocation has been examined by looking at the translocation time for each branch obtained from the IFTP theory and MD simulations. Again the results of the IFTP theory are in excellent agreement with the MD simulations. We have also analytically derived scaling forms for the average translocation time from the IFTP theory showing explicitly how the two branches are dynamically coupled. For both branches the effective translocation exponent, $\alpha$, which is defined as $\tau \sim N_{01}^{\alpha}$ is between 1 and 2. While $\alpha$ for the short branch depends on $N_{01}$ (see Eq. (13)), for the long branch the translocation exponent depends on the values of both contour lengths $N_{01}$ and $N_{02}$ (see Eq. (14)).

Finally, we have used MD simulations to characterise the velocities of the individual monomers for both branches, as well as the probability distribution of the translocation time. As the system becomes more asymmetrical, i.e. one branch gets shorter while the other one becomes longer, the probability distributions of the translocation time distributions for the short and long branches separate. Moreover, the width of the distribution becomes narrower for the short branch.

\section{APPENDIX A}

In this Appendix the explicit forms of $\tilde{\mathcal{U}}_{\mathrm{b}}^{\mathrm{I}}$ and $\tilde{\mathcal{V}}_{2}^{\mathrm{I}}$ as functions of $\nu, A_{\nu}, \tilde{R}_{2}, \tilde{f}$, and $\tilde{\phi}$ are written. In the TP1-TP2 stage (Fig. 1(a)) the equations of motion of the location of the tension fronts for branch 1 and branch 2 are similar to each other as

$$
\dot{\tilde{R}}_{1}^{\mathrm{I}, \mathrm{J}, 12}=\tilde{\mathcal{U}}_{1}^{\mathrm{I}, \mathrm{J}} ; \quad \dot{\tilde{R}}_{2}^{\mathrm{I}, \mathrm{J}, 12}=\tilde{\mathcal{U}}_{2}^{\mathrm{I}, \mathrm{J}},
$$

where I stands for different regimes of SSC, SFC and TRC, J denotes different stages of TP1 and TP2, superscript 12 in the left hand side of Eq. (15) means that both the short as well as the long branches are inside the pore,

$$
\begin{gathered}
\mathcal{U}_{1}^{\mathrm{SSC} 1, \mathrm{TP} 1}=\mathcal{U}_{2}^{\mathrm{SSC} 2, \mathrm{TP} 2}=\frac{B\left(\tilde{R}_{1}\right) \tilde{\phi}_{1}}{1-B\left(\tilde{R}_{1}\right)} ; \\
\mathcal{U}_{1}^{\mathrm{SFC} 1, \mathrm{TP} 1}=\mathcal{U}_{2}^{\mathrm{SFC} 2, \mathrm{TP} 2}=\frac{-B\left(\tilde{R}_{1}\right) \tilde{\phi}_{1}^{2} \mathcal{L}_{\mathrm{SFC} 1}}{1+B\left(\tilde{R}_{1}\right) \tilde{\phi}_{1} \mathcal{L}_{\mathrm{SFC} 1}} ; \\
\mathcal{U}_{1}^{\mathrm{TRC} 1, \mathrm{TP} 1}=\mathcal{U}_{2}^{\mathrm{TRC} 2, \mathrm{TP} 2}= \\
=\frac{B\left(\tilde{R}_{1}\right)\left[-\tilde{\phi}_{1}^{2} \mathcal{L}_{\mathrm{TRC} 1}+\tilde{\phi}_{1}-\tilde{\phi}_{1}\left(\tilde{\phi}_{1} \tilde{R}_{1}\right)^{(\nu-1) / \nu}\right]}{1+B\left(\tilde{R}_{1}\right) \tilde{\phi}_{1} \mathcal{L}_{\mathrm{TRC} 1}},
\end{gathered}
$$

with

$$
\begin{aligned}
B\left(\tilde{R}_{1}\right) & =\nu A_{\nu}^{1 / \nu} \tilde{R}_{1}^{(\nu-1) / \nu} ; \\
\tilde{\phi}_{1}(\tilde{t}) & =\tilde{\phi}_{2}(\tilde{t})=\frac{\tilde{f} / 2}{\tilde{R}_{1}+\tilde{\eta}_{\mathrm{p} 12}+\tilde{s}_{1}}, \\
\mathcal{L}_{\mathrm{SFC} 1} & =\frac{\nu-1}{(2 \nu-1)\left[\tilde{R}_{1}+\tilde{s}_{1}+\tilde{\eta}_{\mathrm{p} 12}\right] \tilde{\phi}_{1}^{2}}-\frac{1}{\tilde{\phi}_{1}} \\
\mathcal{L}_{\mathrm{TRC} 1} & =\frac{\tilde{\phi}_{1}^{-(1+\nu) / \nu} \tilde{R}_{1}^{(\nu-1) / \nu}}{\tilde{R}_{1}+\tilde{s}_{1}+\tilde{\eta}_{\mathrm{p} 12}}\left[\frac{\nu-1}{2 \nu-1} \tilde{\phi}_{1} \tilde{R}_{1}-\tilde{f} / 2\right] .
\end{aligned}
$$



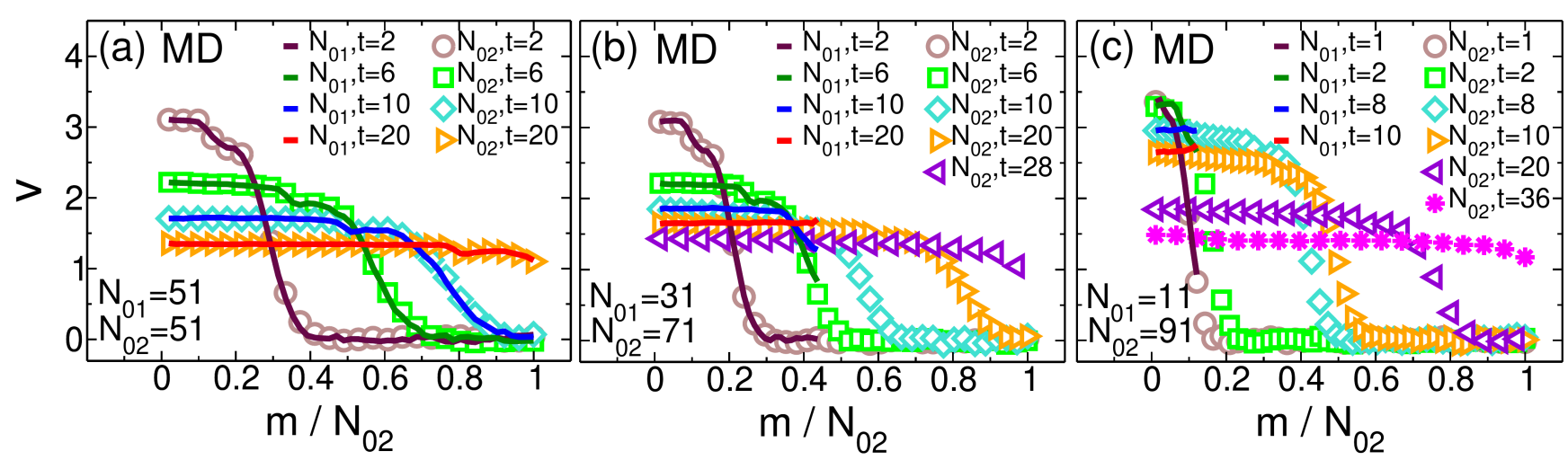

FIG. 4: (a) The velocities of the individual monomers from MD simulations as a function of the normalized monomer index, $m / N_{02}$, at different moments $t=2-20$ for the symmetric folded chain with $N_{01}=N_{02}=51$. Open symbols show the velocities for the branch 2, while the solid lines present the monomer velocities of the branch 1. Panels (b) and (c) are the same as panel (a) but for asymmetrical folded polymer chain with branch contour lengths $N_{01}=31$ and $N_{02}=71$, and $N_{01}=11$ and $N_{02}=91$, respectively. In panels (b) and (c) the label of the horizontal axis has been normalized to the contour length of the longer branch $N_{02}$, and the data is presented during $t=2-28$ and $t=1-36$, respectively. All data are from MD.
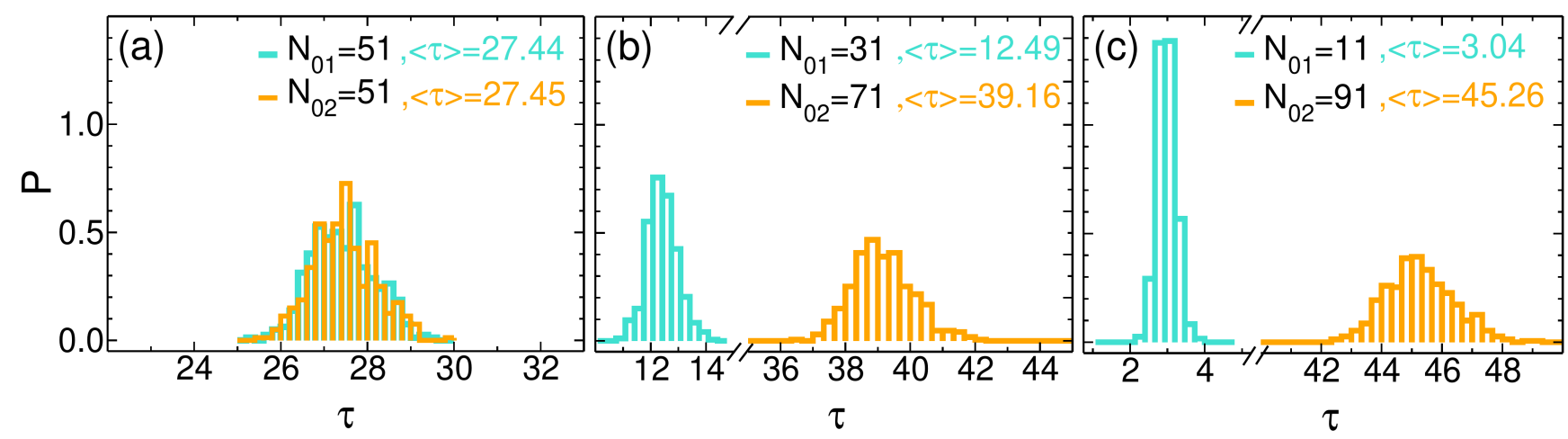

FIG. 5: (a) The probability distribution of the translocation time, $P(\tau)$, for both branches of a folded polymer chain with total contour length of $N_{0}=101$, as a function of the translocation time $\tau$ when the folding is symmetric and the contour length of both branches are the same as $N_{01}=N_{02}=51$. Panels (b) and (c) are the same as panel (a) but for asymmetrical folded polymer with different sets for the contour lengths of the branches $N_{01}=31$ and $N_{02}=71$, and $N_{01}=11$ and $N_{02}=91$, respectively. All data are from $\mathrm{MD}$.

In the PP1-TP2 stage (Fig. 1)(b)) the equations of motion for the tension front location for branch 1 and branch 2, in the SSC1-SSC2 regime are written as

$\dot{\tilde{R}}_{1}^{\mathrm{SSC} 1, \mathrm{PP} 1,12}=-\tilde{\phi}_{1} ; \quad \dot{\tilde{R}}_{2}^{\mathrm{SSC} 2, \mathrm{TP} 2,12}=\frac{B\left(\tilde{R}_{2}\right) \tilde{\phi}_{2}}{1-B\left(\tilde{R}_{2}\right)}$

where

$$
\tilde{\phi}_{1}=\tilde{\phi}_{2}=\tilde{\phi}=\frac{\tilde{f}}{\tilde{R}_{1}+\tilde{R}_{2}+\tilde{s}_{1}+\tilde{s}_{2}+2 \tilde{\eta}_{\mathrm{p} 12}}
$$

is the monomer flux when both branches are inside the nanopore.

For the PP1-TP2 stage (Fig. 1(b)) in the SSC1-SFC2 regime the equations of motion for $\tilde{R}_{1}$ and $\tilde{R}_{2}$ are coupled to each other as

$\dot{\tilde{R}}_{1}^{\mathrm{SSC} 1, \mathrm{PP} 1,12}=-\tilde{\phi} ; \quad \dot{\tilde{R}}_{1}^{\mathrm{SSC} 1, \mathrm{PP} 1,12}+G_{2} \dot{\tilde{R}}_{2}^{\mathrm{SFC} 2, \mathrm{TP} 2,12}=H_{2}$,

in the $\mathrm{SSC} 1-\mathrm{TRC} 2$ regime they are

$\dot{\tilde{R}}_{1}^{\mathrm{SSC} 1, \mathrm{PP} 1,12}=-\tilde{\phi} ; \dot{\tilde{R}}_{1}^{\mathrm{SSC} 1, \mathrm{PP} 1,12}+\mathbb{G}_{2} \dot{\tilde{R}}_{2}^{\mathrm{TRC} 2, \mathrm{TP} 2,12}=\mathbb{H}_{2}$,

in the SFC1-SSC2 regime

$$
\begin{aligned}
\dot{\tilde{R}}_{1}^{\mathrm{SFC} 1, \mathrm{PP} 1,12}+\dot{\tilde{R}}_{2}^{\mathrm{SSC} 2, \mathrm{TP} 2,12} \frac{\mathcal{F}-1}{\mathcal{F}} & =\frac{(1-2 \mathcal{F}) \tilde{\phi}}{\mathcal{F}} \\
\dot{\tilde{R}}_{2}^{\mathrm{SSC} 2, \mathrm{TP} 2,12} & =\frac{B\left(\tilde{R}_{2}\right) \tilde{\phi}}{1-B\left(\tilde{R}_{2}\right)}
\end{aligned}
$$


in the SFC1-SFC2 regime

$$
\begin{aligned}
\dot{\tilde{R}}_{1}^{\mathrm{SFC} 1, \mathrm{PP} 1,12}+\dot{\tilde{R}}_{2}^{\mathrm{SFC} 2, \mathrm{TP} 2,12} \frac{\mathcal{F}-1}{\mathcal{F}} & =\frac{(1-2 \mathcal{F}) \tilde{\phi}}{\mathcal{F}} \\
\dot{\tilde{R}}_{1}^{\mathrm{SFC} 1, \mathrm{PP} 1,12}+\dot{\tilde{R}}_{2}^{\mathrm{SFC} 2, \mathrm{TP} 2,12} G_{2} & =H_{2}
\end{aligned}
$$

in the SFC1-TRC2 regime

$$
\begin{aligned}
\dot{\tilde{R}}_{1}^{\mathrm{SFC} 1, \mathrm{PP} 1,12}+\dot{\tilde{R}}_{2}^{\mathrm{TRC} 2, \mathrm{TP} 2,12} \frac{\mathcal{F}-1}{\mathcal{F}} & =\frac{(1-2 \mathcal{F}) \tilde{\phi}}{\mathcal{F}} \\
\dot{\tilde{R}}_{1}^{\mathrm{SFC} 1, \mathrm{PP} 1,12}+\dot{\tilde{R}}_{2}^{\mathrm{TRC} 2, \mathrm{TP} 2,12} \mathbb{G}_{2} & =\mathbb{H}_{2},
\end{aligned}
$$

in the TRC1-SSC2 regime

$$
\begin{aligned}
\dot{\tilde{R}}_{1}^{\mathrm{TRC} 1, \mathrm{PP} 1,12}+\dot{\tilde{R}}_{2}^{\mathrm{SSC} 2, \mathrm{TP} 2,12} G_{1} & =H_{1} ; \\
\dot{\tilde{R}}_{2}^{\mathrm{SSC} 2, \mathrm{TP} 2,12} & =\frac{B\left(\tilde{R}_{2}\right) \tilde{\phi}}{1-B\left(\tilde{R}_{2}\right)},
\end{aligned}
$$

in the TRC1-SFC2 regime

$$
\begin{aligned}
& \dot{\tilde{R}}_{1}^{\mathrm{TRC} 1, \mathrm{PP} 1,12}+\dot{\tilde{R}}_{2}^{\mathrm{SFC} 2, \mathrm{TP} 2,12} G_{1}=H_{1} ; \\
& \dot{\tilde{R}}_{1}^{\mathrm{TRC} 1, \mathrm{PP} 1,12}+\dot{\tilde{R}}_{2}^{\mathrm{SFC} 2, \mathrm{TP} 2,12} G_{2}=H_{2},
\end{aligned}
$$

and in the TRC1-TRC2 regime

$$
\begin{aligned}
& \dot{\tilde{R}}_{1}^{\mathrm{TRC} 1, \mathrm{PP} 1,12}+\dot{\tilde{R}}_{2}^{\mathrm{TRC} 2, \mathrm{TP} 2,12} G_{1}=H_{1} ; \\
& \dot{\tilde{R}}_{1}^{\mathrm{TRC} 1, \mathrm{PP} 1,12}+\dot{\tilde{R}}_{2}^{\mathrm{TRC} 2, \mathrm{TP} 2,12} \mathbb{G}_{2}=\mathbb{H}_{2},
\end{aligned}
$$

where

$$
\begin{aligned}
G_{\mathrm{b}} & =\frac{B\left(\tilde{R}_{\mathrm{b}}\right) \mathcal{F}-1}{B\left(\tilde{R}_{\mathrm{b}}\right)(\mathcal{F}-1)} ; \\
H_{\mathrm{b}} & =\frac{B\left(\tilde{R}_{\mathrm{b}}\right)(1-2 \mathcal{F}) \tilde{\phi}}{B\left(\tilde{R}_{\mathrm{b}}\right)(\mathcal{F}-1)} ; \\
\mathbb{G}_{2} & =\frac{1+(\mathcal{F}-1) \tilde{\phi} \tilde{R}_{2}-B^{-1}\left(\tilde{R}_{2}\right)\left(\tilde{\phi} \tilde{R}_{2}\right)^{(1-\nu) / \nu}}{(\mathcal{F}-1) \tilde{\phi} \tilde{R}_{2}} ; \\
\mathbb{H}_{2} & =\frac{2 \tilde{\phi}(1-\mathcal{F}) \tilde{\phi} \tilde{R}_{2}-\tilde{\phi}\left(\tilde{\phi} \tilde{R}_{2}\right)^{(1-\nu) / \nu}}{(\mathcal{F}-1) \tilde{\phi} \tilde{R}_{2}} \\
\mathcal{F} & =1+\frac{1-\nu}{2 \nu-1} \frac{1}{\tilde{f}} .
\end{aligned}
$$

In the $\tau_{1}$-TP2 stage for the $\mathrm{SSC} 2, \mathrm{SFC} 2$ and $\mathrm{TRC} 2$ regimes (Fig. 1(c)), wherein the translocation process for the shorter branch has been completed and only the longer branch is inside the nanopore, the equations of motion for $\tilde{R}_{2}$ are obtained as

$$
\begin{aligned}
& \dot{\tilde{R}}_{2}^{\mathrm{SSC} 2, \mathrm{TP} 2,2}=\frac{B\left(\tilde{R}_{2}\right) \tilde{\phi}_{2}}{1-B\left(\tilde{R}_{2}\right)} ; \\
& \dot{\tilde{R}}_{2}^{\mathrm{SFC} 2, \mathrm{TP} 2,2}=\frac{B\left(\tilde{R}_{2}\right) \tilde{\phi}_{2} \mathcal{F}}{1-B\left(\tilde{R}_{2}\right) \mathcal{F}} ; \\
& \dot{\tilde{R}}_{2}^{\mathrm{TRC} 2, \mathrm{TP} 2,2}= \\
& \quad=\frac{B\left(\tilde{R}_{2}\right)\left[-\tilde{\phi}_{2}\left(\tilde{\phi}_{2} \tilde{R}_{2}\right)^{(2 \nu-1) / \nu}(1-\mathcal{F})+\tilde{\phi}\right]}{1+B\left(\tilde{R}_{2}\right)\left(\tilde{\phi}_{2} \tilde{R}_{2}\right)^{(\nu-1) / \nu}\left[\tilde{\phi}_{2} \tilde{R}_{2}(1-\mathcal{F})-1\right]},
\end{aligned}
$$

while in the $\tau_{1}$-PP2 stage (Fig. 1(d)) the equations of motion for $\tilde{R}_{2}$ are

$$
\begin{aligned}
\dot{\tilde{R}}_{2}^{\mathrm{SSC} 2, \mathrm{PP} 2,2} & =-\tilde{\phi}_{2} ; \\
\dot{\tilde{R}}_{2}^{\mathrm{SFC} 2, \mathrm{PP} 2,2} & =-\tilde{\phi}_{2} ; \\
\dot{\tilde{R}}_{2}^{\mathrm{TRC} 2, \mathrm{PP} 2,2} & =\frac{-\left(\tilde{\phi}_{2} \tilde{R}_{2}\right)^{(2 \nu-1) / \nu}(1-\mathcal{F})+\tilde{\phi}}{\left(\tilde{\phi}_{2} \tilde{R}_{2}\right)^{(\nu-1) / \nu\left[\tilde{\phi}_{2} \tilde{R}_{2}(1-\mathcal{F})-1\right]}} .
\end{aligned}
$$

In the $\tau_{1}$-TP2 and $\tau_{1}$-PP2 stages the monomer flux is

$$
\tilde{\phi}_{2}=\frac{\tilde{f}}{\tilde{R}_{2}+\tilde{s}_{2}+N_{01}+\tilde{\eta}_{\mathrm{p} 2}} .
$$

Finally, it should be mentioned that in order to obtain the time evolution of the tension fronts, in the left hand side of the above equations of motion the following replacement must be done $\dot{\tilde{R}}_{1}^{\mathrm{I}, \mathrm{J}, 12}=\dot{\tilde{R}}_{1}, \dot{\tilde{R}}_{2}^{\mathrm{I}, \mathrm{J}, 12}=\dot{\tilde{R}}_{2}$. In the equations $\tilde{R}_{1}$ and $\tilde{R}_{2}$ present the time evolution of the tension front in the corresponding regimes and stages mentioned by superscripts.

\section{Acknowledgments}

S.C. and B.G. acknowledge DAE-BRNS (37(2)/ 14/08/2016-BRNS/37022) for computer facilities. B.G. acknowledges DAE-BRNS (37(2)/ 14/08/2016BRNS/37022) and IISER Pune for fellowship. T.A-N. has been supported in part by the Academy of Finland through its PolyDyna (no. 307806) and QFT Center of Excellence Program grants (no. 312298). J.S. thanks Takahiro Sakaue for enlightening discussions.
[1] M. Akeson, D. Branton, J.J. Kasianowicz, E. Brandin and D.W. Deamer, Biophys. J. 77, 3227 (1999).

[2] V.R. Lingappa, J. Chaidez, C.S. Yost and J. Hedgpeth, Proc. Natl. Acad. Sci. 81, 456 (1984).

[3] S.W.P. Turner, M. Cabodi and H.G. Craighead, Phys. Rev. Lett. 88, 128103 (2002).

[4] D.C. Chang, Guide to Electroporation and Electrofusion
(Academic: New York, 1992).

[5] J.J. Kasianowicz, E. Brandin, D. Branton and D.W. Deamer, Proc. Natl. Acad. Sci. 93, 13770 (1996).

[6] A. Meller, L. Nivon and D. Branton, Phys. Rev. Lett. 86, 3435 (2001).

[7] W. Sung and P.J. Park, Phys. Rev. Lett. 77, 783 (1996).

[8] M. Muthukumar, J. Chem. Phys. 111, 10371 (1999). 
[9] M. Muthukumar, J. Chem. Phys. 118, 5174 (2003).

[10] T. Sakaue, Phys. Rev. E 76, 021803 (2007).

[11] T. Sakaue, Phys. Rev. E 81, 041808 (2010).

[12] T. Saito and T. Sakaue, Eur. Phys. J. E 34, 135 (2012).

[13] T. Ikonen, A. Bhattacharya, T. Ala-Nissila and W. Sung, Phys. Rev. E 85, 051803 (2012).

[14] T. Ikonen, A. Bhattacharya, T. Ala-Nissila and W. Sung, J. Chem. Phys. 137, 085101 (2012).

[15] T. Ikonen, A. Bhattacharya, T. Ala-Nissila and W. Sung, Europhys. Lett. 103, 38001 (2013).

[16] T. Ikonen, J. Shin, W. Sung and T. Ala-Nissila, J.Chem. Phys. 136, 205104 (2012).

[17] V.V. Palyulin, T. Ala-Nissila and R. Metzler, Soft Matter 10, 9016 (2014).

[18] J. Sarabadani, T. Ikonen and T. Ala-Nissila, J. Chem. Phys. 141, 214907 (2014).

[19] J. Sarabadani, T. Ikonen and T. Ala-Nissila, J. Chem. Phys. 143, 074905 (2015).

[20] J. Sarabadani, B. Ghosh, S. Chaudhury and T. AlaNissila, Europhys. Lett. 120, 38004 (2017).

[21] J. Sarabadani, T. Ikonen, H. Mökkönen, T. Ala-Nissila, S. Carson, M. Wanunu, Sci. Rep. 7, 7423 (2017).

[22] J. Sarabadani and T. Ala-Nissila, J. Phys. Condens. Matter 30, 274002 (2018).

[23] H.W. de Haan and G.W. Slater, Phys. Rev. E 81, 051802 (2010).

[24] H.W. de Haan and G.W. Slater, J. Chem. Phys. 136, 204902 (2012).

[25] M.G. Gauthier and G.W. Slater, Phys. Rev. E 79, 021802 (2009).

[26] J.A. Cohen, A. Chaudhuri and R. Golestanian, J. Chem. Phys. 107, 238102 (2011).

[27] J.A. Cohen, A. Chaudhuri and R. Golestanian, Phys. Rev. X 2, 021002 (2012).

[28] J.A. Cohen, A. Chaudhuri and R. Golestanian, J. Chem.
Phys. 137, 204911 (2012).

[29] K. Luo, T. Ala-Nissila, S.-C. Ying and A. Bhattacharya, Phys. Rev. Rett. 100, 058101 (2008).

[30] G. Sigalov, J. Comer, G. Timp and A. Aksimentiev, Nano Lett. 8, 56 (2008).

[31] A. Milchev, J. Phys. Condens. Matter 23, 103101 (2011).

[32] J. Mathe, A. Aksimentiev, AD. R. Nelson, K. Schulten and A. Meller, Proc. Natl. Acad. Sci. 102, 12377 (2005).

[33] C. Dekker, Nature Nanotechnology 2, 209 (2007).

[34] A.J. Storm, J.H. Chen, X.S. Ling, H.W. Zandbergen and C. Dekker, Nature Materials 2, 537 (2003).

[35] U.F. Keyser, J. van der Does, C. Dekker and N.H. Dekker, Rev. Sci. Instruments 77, 105105 (2006).

[36] J.B. Heng, A. Aksimentiev, C. Ho, P. Marks, Y.V. Grinkova, S. Sligar, K. Schulten and G. Timp, Nanao Lett. 5, 1883 (2005).

[37] J. Nakane, M. Akeson and A. Marziali, Electrophoresis 23, 2592 (2002).

[38] J.J. Kasianowicz, S.E. Henrickson, H.H. Weetall and B. Robertson, Nature 73, 2268 (2001).

[39] H. Bayley and P.S. Cremer, Nature 413, 226 (2001).

[40] H. Bayley and C.R. Martin, Chem. Rev. 100, 2575 (2000).

[41] A.J. Storm, J.H. Chen, H.W. Zandbergen, J.-F. Joanny and C. Dekker, Nano Lett. 5, 1193 (2005).

[42] A.J. Storm, J.H. Chen, H.W. Zandbergen and C. Dekker, Phys. Rev. E 71, 051903 (2005).

[43] C. Forrey and M. Muthukumar, J. Chem. Phys. 127, 015102 (2007).

[44] S. Kotsev and A.B. Kolomeisky, J. Chem. Phys. 127, 185103 (2007).

[45] P. Rowghanian and A.Y. Grosberg, J. Phys. Chem. B 115, 14127 (2011).

[46] S. Plimpton, J. Comp. Phys. 117, 1 (1995). 